\section{Positioning the breathing but unresponsive patient: what is the evidence?}

\author{
Robert C Tasker ${ }^{1,2}$
}

First aid for the unconscious but still breathing infant or child who has no other life-threatening condition is something everyone needs to know. Providing spinal injury is not suspected, first aid care involves placing the subject in the recovery position so that the airway remains clear and open. ${ }^{1}$ Videos that explain and show how to position such an infant or a child can be found on a number of professional websites, including National Health Service Choices, ${ }^{1}$ British Red Cross, ${ }^{2}$ St. John Ambulance ${ }^{3}$ and the Epilepsy Society. ${ }^{4}$

A good quality clinical practice guideline (CPG) is based on a process that includes defining a clear PICO (Patient, Intervention, Comparison, Outcome) question, seeking the highest quality evidence available, appraising and synthesising this evidence, then creating a recommendation by assimilating this information with the clinical/patient expertise of the CPG developers. Recently, the use of the lateral, side-lying recovery position in the breathing but unresponsive patient was reviewed and published by the International Liaison Committee on Resuscitation (ILCOR) and American Heart Association (AHA). ${ }^{5}$ Over the timeline of ILCOR's scientific evidence evaluation and review (April 2013 to February 2015), before publication on 15 October 2015, only a few small studies and, at best, low-quality evidence were identified in the literature for outcomes such as 'incidence of aspiration', 'need for airway management', 'the likelihood of cervical spinal injury' and 'complications'. ${ }^{6}$ The reviewers found no evidence to address the critical outcomes of 'overall mortality' or 'incidence of cardiac arrest'. 6 Also, there were no randomised controlled trials (RCTs). In the new

${ }^{1}$ Division of Critical Care Medicine, Departments of Neurology, Anesthesiology, Perioperative and Pain Medicine, Boston Children's Hospital, Boston, USA; ${ }^{2}$ Departments of Neurology and Anesthesiology, Harvard Medical School, Boston, USA

Correspondence to Professor Robert C Tasker, Division of Critical Care Medicine, Department of Neurology, Department of Anesthesiology, Perioperative and Pain Medicine, Boston Children's Hospital, Boston MA 02115, USA; robert.tasker@childrens.harvard.edu guidelines, readers will see that an updated version of the Grades of Recommendation, Assessment, Development, and Evaluation development tool (http://www. guidelinedevelopment.org) was used for applying level (ie, quality) of evidence and class (ie, strength) of recommendation. ${ }^{7}$ The experts considered evidence for the recovery position by first aid providers was in the category of 'weak', which means better quality evidence has the potential to overturn their conclusion, rather than that any recommendations should be ignored. In fact, the experts' values and preferences statement placed high value on the importance of decreasing the risk of aspiration or the need for more advanced airway management. And, so, they concluded, "Given the absence of high quality evidence, we (ILCOR) are suggesting use of a recovery position due to the lack of demonstrated associated risk." 5

Julliand et $a l^{8}$ report findings from a prospective, observational, multicentre European study of consecutive children seen in 11 paediatric emergency departments (EDs) because of a period of unconsciousness, either at the time of presentation or in the previous $24 \mathrm{~h}$. This is an important study, which was carried out over three months, that evaluated whether primary care givers used the recovery position manoeuvre and whether this intervention was associated with decreased hospital admission rate of these children. There were 557 children in the study, and they represented $5.5 \%$ of all paediatric ED consultations. Half of the cases were post-ictal (febrile and nonfebrile seizure in 147 and 131 cases, respectively) and a further $22.4 \%$ were seen after vasovagal syncope. In all, the recovery position was used in $26.2 \%$ of cases. In another $53 \%$ of cases, some other manoeuvre was used-most notable was the potentially dangerous use of shaking in 91 cases (16.5\%). Discharge home after visiting the ED occurred in $43.8 \%$ of cases. The primary analysis showed an independent association between use of the recovery position and a decreased admission rate with an adjusted OR 0.28 (95\% CI 0.17 to 0.48 , $\mathrm{p}<000.1)$. Dangerous manoeuvres were independently associated with an increased rate of admission with an adjusted OR of 2.12 (95\% CI 1.19 to $3.78, \mathrm{p}=0.01$ )

This is the largest paediatric study in the literature, but does it provide new evidence that will change the strength of recommendation from ILCOR for using the recovery position? The current report had defined inclusion and exclusion criteria but it was not an RCT. The reality, however, is that only observational studies are feasible in emergency, first-responder care, and it is unlikely that an RCT on this intervention will ever be undertaken. In the current report, ${ }^{8}$ the authors have a number of individuals that were placed in the recovery position, and others that were not. Because it is not clear how patients receiving the intervention differed from those not undergoing placement in the recovery position, it is not possible to examine causation (ie, whether the intervention resulted in better outcomes). Acknowledging this well-known limitation of observational studies, the key question, then, is what conclusions can be drawn about the first aid recovery position in the unresponsive but breathing patient based on the findings of the study reported by Julliand et al? ${ }^{8}$

In translating the information from the current report into priorities in first aid, notable features of the cohort must be considered. Nearly $40 \%$ of these patients had had a previous episode of loss of consciousness. Nearly one-half of the patients had epilepsy. Almost one-third of the patients had an episode of unconsciousness lasting $2 \mathrm{~min}$ or longer. So, is this new information really about using the recovery position in patients with known seizure disorder? And, if so, wouldn't such patients be discharged from the ED once recovery has occurred? Alternatively, is it about the treating physician's behaviour when presented with parents who did not report using the recovery position and therefore admitting the child in order to provide support and education? Thus, it is unclear whether the associations reported merely reflect another factor and the practice of using the recovery position manoeuvre because of prior experience/education of parents in a population of children with a selflimiting condition that does not usually warrant admission. Since it is unlikely that an RCT on using the recovery position in the breathing but unresponsive patient will be carried out, the new data reported by Julliand et $a l^{8}$ fill an important gap in our knowledge, in paediatric 
emergency practice. It not only supports what has been recommended recently, ${ }^{5} 6$ but also adds to the body of research that remains potentially biased. However, these biases are unlikely to disguise any harms from the recovery position and so it remains the case that its benefit far outweighs any harms.

Taken together, the report by Julliand et $a l^{8}$ provides new data to inform clinical care, but do we need to wait until 2020 for another 5-year cycle of 'batch-and-queue' of such papers before the 2015 AHA and ILCOR guidelines are updated and disseminated? We do not. The recently published 2015 cycle $^{9}$ also marks the transition to a continuous evidence review process, with an online, living website that will be updated as ILCOR completes evidence reviews (see http://www.ilcor.org/seers). ${ }^{9}$ It is not Archives of Disease in Childhood's role to decide what should or shouldn't be incorporated into the AHA/ILCOR evidence and recommendations, but the new system does mean that we are now in an era in which papers, such as the current report from a multicentre ED study, ${ }^{8}$ will rightly be reviewed and evaluated for contemporary practitioners.

Competing interests None declared.

Provenance and peer review Commissioned; internally peer reviewed.

To cite Tasker RC. Arch Dis Child 2016;101:508509.

Received 22 October 2015

Revised 1 February 2016

Accepted 2 February 2016

Published Online First 23 February 2016

\section{SLinked}

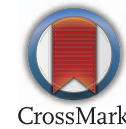

- http://dx.doi.org/10.1136/archdischild-2015-308857

Arch Dis Child 2016;101:508-509.

doi:10.1136/archdischild-2015-309362

\section{REFERENCES}

1 First aid-recovery position. NHS Choices: your health, your choices. http://www.nhs.uk/Conditions/ Accidents-and-first-aid/Pages/The-recovery-position. aspx (accessed Jan 2016).

2 Everyday First Aid: first aid for someone who's unconscious and breathing. British Red Cross. http:// www.redcross.org.uk/What-we-do/First-aid/
Everyday-First-Aid/Unconscious-and-breathing (accessed Jan 2016).

3 The Recovery Position. St John Ambulance. http:/l www.sja.org.uk/sja/first-aid-advice/first-aid-techniques/ the-recovery-position.aspx (accessed Jan 2016).

4 Step-by-step recovery position. Epilepsy Society. http:// www.epilepsysociety.org.uk/step-step-recoveryposition\#.VhqeUOud8Y8 (accessed Jan 2016).

5 Singletary EM, Charlton NP, Epstein JL, et al. Part 15: first aid: 2015 American Heart Association and American Red Cross Guidelines Update for First Aid. Circulation 2015;132(Suppl 2):S574-89.

6 Recovery Position. International Liaison Committee On Resuscitation Scientific Evidence Evaluation and Review System. https://volunteer.heart.org/apps/pico/ Pages/PublicComment.aspx?q=517 (accessed Jan 2016).

7 Morrison LJ, Gent LM, Lang E, et al. Part 2: evidence evaluation and management of conflicts of interest: 2015 American Heart Association Guidelines Update for Cardiopulmonary Resuscitation and Emergency Cardiovascular Care. Circulation 2015;132(Suppl 2): S368-82.

8 Julliand S, Desmarest M, Gonzalez L, et al. Recovery position is significantly associated with a reduced admission rate of children with loss of consciousness. Arch Dis Child 2016;101:519-24.

9 Neumar RW, Shuster M, Callaway CW, et al. Part 1: executive summary: 2015 American Heart Association Guidelines Update for Cardiopulmonary Resuscitation and Emergency Cardiovascular Care. Circulation 2015;132(Suppl 2):S315-67. 